\title{
ASYMPTOTIC EXPANSIONS IN A NONHOMOGENEOUS DIFFERENTIAL EQUATION
}

THOMAS G. HALLAM

In recent papers [2]-[8] asymptotic behavior of the solutions of an ordinary differential equation has been discussed by the development of an asymptotic expansion for the solution that is valid to one term. In [5], [7], asymptotic expansions valid to two terms were obtained for certain solutions of a differential equation. These results which were valid to two terms were derived under slightly stronger conditions than those results which were valid for a single term. In this note, under an appropriate strengthening of the hypothesis which yields an asymptotic expansion valid to one term, we will show that an asymptotic expansion of solutions of a nonhomogeneous nonlinear differential equation may be developed which is valid to $N<\infty$ terms. The method used here differs from those in [1] to the extent that a singular integral equation is not used.

We consider, as in [3], the nonhomogeneous differential equation

$$
u^{(n)}+f\left(t, u, u^{(1)}, \cdots, u^{(n-1)}\right)=h(t), \quad u^{(j)}=d^{j} u / d t^{j},
$$

where

$$
\left|f\left(t, u, u^{(1)}, \cdots, u^{(n-1)}\right)\right| \leqq \sum_{i=0}^{n-1} g_{i}(t)\left|u^{(i)}\right| r_{i}
$$

with

(2a) $r_{i}>0, i=0,1, \cdots, n-1$;

(2b) $g_{i}(t)$ are continuous for $t \geqq t_{0}$; and furthermore, if $g_{i}(t) \not \equiv 0$, then there exists a $p_{i}$ such that $\lim _{t \rightarrow \infty} t^{p_{i}} g_{i}(t)=c_{i} \neq 0$ for $i=0,1, \cdots$, $n-1$.

(3) $h(t)=b_{0} t^{m}+R(t)$, with

(3a) $R(t)=o\left(t^{m}\right)$ as $t \rightarrow \infty$;

(3b) $m>-1$ and $b_{0} \neq 0$.

Always, $f$ and $h$ are continuous functions for $t \geqq t_{0}$ and all $u$, $u^{(1)}, \cdots, u^{(n-1)}$. In previous terminology [3], [4] the forcing term is said to have primary part $t^{m}, m>-1$.

THEOREM. Let conditions (2) and (3) be satisfied; furthermore, suppose

$$
t^{-m} h(t)=b_{0}+b_{1} t^{-1}+b_{2} t^{-2}+\cdots+b_{N} t^{N}+R_{N}(t)
$$

Presented to the Society, January 27, 1967; received by the editors June 10, 1966. 
where $R_{N}(t)=o\left(t^{-N}\right)$ as $t \rightarrow \infty$. If $m>N-1$ and

$$
p_{i}>r_{i}(m+n-i)-m+N
$$

for all $i=0,1, \cdots, n-1$, then there exist solutions $u(t)$ of (1) which possess the asymptotic expansion

$$
\frac{u^{(k)}(t)}{t^{m+n-k}} \sim a_{0}^{(k)}+a_{1}^{(k)} t^{-1}+a_{2}^{(k)} t^{-2}+\cdots+a_{N}^{(k)} t^{-N}
$$

where

$$
a_{j}^{(k)}=\frac{b_{j}}{(m+1-j)(m+2-j) \cdots(m+n-k-j)},
$$

$k=0,1, \cdots, n-1, j=0,1, \cdots, N$. If $r=\max _{i} r_{i}$, then $r \leqq 1$ implies all solutions of (1) possess the asymptotic expansion (4).

Proof. In [3], it was established that $\sum_{k=0}^{n-1}\left|u^{(k)}(t) / t^{m+n-k}\right|$ is bounded provided $m>-1$ and $p_{i}>r_{i}(m+n-i)-m, i=0,1, \cdots$, $n-1$. Under these conditions, the asymptotic expansion to one term

$$
\frac{u^{(k)}(t)}{t^{m+n-k}} \sim a_{0}^{(k)} \neq 0, \quad k=0,1, \cdots, n-1,
$$

was determined where the $a_{0}^{(\mathbf{k})}$ were given as

$$
\boldsymbol{a}_{0}^{(k)}=\frac{b_{0}}{(m+n-k)(m+n-k-1) \cdots(m+1)} .
$$

The conditions concerning the size of $m$ and $p_{i}$ are sufficient to have the asymptotic dependence of the solutions of equation (1) entirely upon the forcing term; that is, it will be shown that the asymptotic expansion of the solution depends implicitly upon the asymptotic expansion of the forcing term $h(t)$.

Integrating (1) $n-k$ times gives for $t \geqq t_{0} \geqq 1$,

$$
\begin{aligned}
u^{(k)}(t)= & \theta_{0}+\theta_{1} t+\theta_{2} t^{2}+\cdots+\theta_{n-k-1} t^{n-k-1} \\
& +\int_{t_{0}}^{t} \frac{(t-s)^{n-k-1}}{(n-k-1) !} h(s) d s \\
& -\int_{t_{0}}^{t} \frac{(t-s)^{n-k-1}}{(n-k-1) !} f\left(s, u(s), u^{(1)}(s), \cdots, u^{(n-1)}(s)\right) d s .
\end{aligned}
$$

It will now be shown that for any solution $u(t)$ of equation (1) such that $\sum_{i=0}^{n-1}\left|u^{(i)}(t) / t^{m+n-i}\right|$ is bounded then such $u(t)$ possess the given asymptotic expansion (4). It was shown in [3] that 


$$
\sum_{i=0}^{n-1}\left|u^{(i)}(t) / t^{m+n-i}\right|
$$

is always bounded under our hypothesis provided $r \leqq 1$. If $r>1$, then the existence of solutions $u(t)$ of (1) such that

$$
\sum_{i=0}^{n-1}\left|u^{(i)}(t) / t^{m+n-i}\right|
$$

is bounded was demonstrated.

We wish to evaluate

$$
\lim _{t \rightarrow \infty} t^{N}\left[\frac{u^{(k)}(t)}{t^{m+n-k}}-a_{0}^{(k)}-a_{1}^{(k)} t^{-1}-\cdots-a_{N-1}^{(k)} t^{-(N-1)}\right]
$$

where $k=0,1, \cdots, n-1, a_{f}^{(\boldsymbol{k})}$ are suitably chosen, and $u^{(k)}(t)$ is given by (5). In order to establish the existence of this limit, we first note that the following limits hold.

$$
\begin{gathered}
\lim _{t \rightarrow \infty}\left[\theta_{0}+\theta_{1} t+\theta_{2} t^{2}+\cdots+\theta_{n-k-1} t^{n-k-1}\right] t^{k-m-n+N}=0, \\
k=0,1, \cdots, n-1 . \\
\lim _{t \rightarrow \infty}\left[t^{k-m-n+N} \int_{t_{0}}^{t} \frac{(t-s)^{n-k-1}}{(n-k-1) !}\right. \\
\left.\cdot f\left(s, u(s), u^{(1)}(s), \cdots, u^{(n-1)}(s)\right) d s\right]=0, \\
k=0,1, \cdots, n-1 .
\end{gathered}
$$

In (7), the term that will be dominant in the asymptotic expansion is $\theta_{n-k-1} t^{N-m-1}$; thus, noting $m>N-1$, equality ( 7$)$ is established.

For the determination of (8), the following inequality will be needed.

$$
\begin{aligned}
\mid t^{k-m-n+N} & \int_{t_{0}}^{t} \frac{(t-s)^{n-k-1}}{(n-k-1) !} f\left(s, u(s), u^{(1)}(s), \cdots, u^{(n-1)}(s)\right) d s \mid \\
& \leqq t^{N-m-1} \int_{t_{0}}^{t} \sum_{i=0}^{n-1} g_{i}(s) s^{p_{i}}\left|\frac{u^{(i)}(s)}{s^{m+n-i}}\right|^{r_{i}} s^{r_{i}(m+n-i)-p_{i}} d s \\
& \leqq \sum_{i=0}^{N-1} B_{i} t^{N-m-1} \int_{t_{0}}^{t} s^{r_{i}(m+n-i)-p_{i}} d s \\
& =t^{N-m-1}\left\{\sum_{\alpha} B_{\alpha}\left[\frac{t^{r_{\alpha}(m+n-\alpha)-p_{\alpha}+1}-t_{0}{ }^{r_{\alpha}(m+n-\alpha)-p_{\alpha}+1}}{r_{\alpha}(m+n-\alpha)-p_{\alpha}+1}\right]\right. \\
& \left.+\sum_{\beta} B_{\beta}\left[\ln t-\ln t_{0}\right]\right\}
\end{aligned}
$$


where the $\alpha(\beta)$ index set has $p_{\alpha}\left(p_{\beta}\right)$ such that

$$
p_{\alpha} \neq r_{\alpha}(m+n-\alpha)+1 \quad\left(p_{\beta}=r_{\beta}(m+n-\beta)+1\right) .
$$

Using $m>N-1$ and

$$
p_{i}>r_{i}(m+n-i)-m+N, \quad i=0,1, \cdots, n-1,
$$

the limit in (8) follows from the above inequality.

Before evaluating the limit in (6), it remains to consider the expression

$$
t^{k-m-n+N} \int_{t_{0}}^{t} \frac{(t-s)^{n-k-1}}{(n-k-1) !} h(s) d s .
$$

Some lemmas will be given which are useful in our considerations.

Lemмa 1. Let $h(t)$ be as given in the theorem and $z$ be any positive integer, then

$$
\begin{aligned}
& \int_{t_{0}}^{t}(t-s)^{z} h(s) d s \\
& =\sum_{r=0}^{z}\left(\begin{array}{l}
z \\
r
\end{array}\right)(-1)^{r}\left\{\sum_{i=0}^{n} \frac{b_{i}}{r+m+1-i} t^{m+z+1-i}\right. \\
& \left.\quad+C_{r}\left(t_{0}\right) t^{z-r}+t^{z-r} \int_{t_{0}}^{t} s^{r+m} R_{N}(s) d s\right\},
\end{aligned}
$$

where $C_{r}\left(t_{0}\right)$ is a constant.

Proof of Lemma 1. The lemma follows by use of the binomial theorem and integration.

Lemma 2. If $m>N-1$ and $j=0,1, \cdots, N$, then for any positive integer $z$,

$$
\begin{aligned}
\sum_{r=0}^{z}\left(\begin{array}{l}
z \\
r
\end{array}\right)(-1)^{r} \frac{1}{r+} & m+1-j \\
= & \frac{z !}{(m+1-j)(m+2-j) \cdots(m+z+1-j)} .
\end{aligned}
$$

Proof of Lemma 2. The lemma is clearly true for $z=1$. We assume that the statement is valid for the integer $z-1$ and consider the following expression. 


$$
\begin{aligned}
\sum_{r=0}^{z}\left(\begin{array}{l}
z \\
r
\end{array}\right)(-1)^{r} & \frac{1}{r+m+1-j} \\
= & \frac{1}{m+1-j}+\sum_{r=1}^{z-1}\left[\left(\begin{array}{c}
z-1 \\
r
\end{array}\right)+\left(\begin{array}{c}
z-1 \\
r-1
\end{array}\right)\right] \\
& \cdot(-1)^{r}\left(\frac{1}{r+m+1-j}\right)+\frac{(-1)^{z}}{z+m+1-j} \\
= & \sum_{r=0}^{z-1}\left(\begin{array}{c}
z-1 \\
r
\end{array}\right)(-1)^{r} \frac{1}{r+m+1-j} \\
& -\sum_{r=0}^{z-1}\left(\begin{array}{c}
z-1 \\
r
\end{array}\right)(-1)^{r} \frac{1}{r+m+2-j} \\
= & z ! /(m+1-j)(m+2-j) \cdots(m+z+1-j) .
\end{aligned}
$$

This completes the proof of Lemma 2.

Thus, using Lemma 1 and 2, we obtain

$$
\begin{aligned}
& t^{k-m-n+N} \int_{t_{0}}^{t} \frac{(t-s)^{n-k-1}}{(n-k-1) !} h(s) d s \frac{1}{(n-k-1) !}\left\{\sum _ { r = 0 } ^ { n - k - 1 } ( \begin{array} { c } 
{ n - k - 1 } \\
{ r }
\end{array} ) ( - 1 ) ^ { r } \left[\sum_{j=0}^{N} \frac{b_{j} t^{N-j}}{r+m+1-j}\right.\right. \\
&\left.\left.\quad+C_{r}\left(t_{0}\right) t^{N-m-r-1}+t^{N-m-r-1} \int_{t}^{t} s^{r+m} R_{N}(s) d s\right]\right\} \\
&=\frac{1}{(n-k-1) !}\left\{\sum_{j=0}^{N} \frac{(n-k-1) ! b_{j} t^{N-j}}{(m+1-j)(m+2-j) \cdots(m+n-k-j)}\right. \\
& \quad+\sum_{r=0}^{n-k-1}\left(\begin{array}{c}
n-1 \\
k-1
\end{array}\right)(-1)^{r} C_{r}\left(t_{0}\right) t^{N-m-r-1} \\
&\left.\quad+\sum_{r=0}^{n-k-1}\left(\begin{array}{c}
n-k-1 \\
r
\end{array}\right)(-1)^{r} t^{N-m-r-1} \int_{t_{0}}^{t} s^{m+r} R_{N}(s) d s\right\} .
\end{aligned}
$$

The limit in (6) will now be evaluated. Observing the limits in (7) and (8), (6) reduces to a consideration of

$$
\begin{aligned}
\lim _{t \rightarrow \infty}\left[t^{k-m-n+N} \int_{t_{0}}^{t} \frac{(t-s)^{n-k-1}}{(n-k-1) !} h(s) d s-a_{0}^{(k)} t^{N}\right. & \\
& \left.\quad-a_{1}^{(k)} t^{N-1}+\cdots-a_{N-1}^{(k)} t\right],
\end{aligned}
$$

where the $a_{f}^{(\mathbf{k})}$ are given by 


$$
\begin{aligned}
& a_{j}^{(k)}=b_{j} /(m+1-j)(m+2-j) \cdots(m+n-k-j), \\
& j=0,1, \cdots, N-1, \quad k=0,1, \cdots, n-1 .
\end{aligned}
$$

An inductive argument will be used to show that the limit in (10) is equal to $a_{N}^{(\mathbf{k})}$ where $a_{N}^{(\boldsymbol{k})}$ is given by (11) evaluated at $j=N$. For $N=0$, the result was established in [3]. We assume that the statement is true for $N-1$ and consider the statement at the integer $N$. Now,

$$
\begin{aligned}
& \lim _{t \rightarrow \infty}\left[t^{k-m-n+N} \int_{t_{0}}^{t} \frac{(t-s)^{n-k-1}}{(n-k-1) !} h(s) d s\right. \\
& \left.-a_{0}^{(k)} t^{N}-a_{1}^{(k)} t^{N-1}-\cdots-a_{N-1}^{(k)} t\right] \\
& =\lim _{t \rightarrow \infty}\left\{\frac{1}{(n-k-1) !}\right. \\
& \cdot\left[\sum_{j=0}^{N} \frac{(n-k-1) ! b_{j} t^{N-}}{(m+1-j)(m+2-j) \cdots(m+n-k-j)}\right. \\
& +\sum_{r=0}^{n-k-1}\left(\begin{array}{c}
n-k-1 \\
r
\end{array}\right)(-1)^{r} C_{r}\left(t_{0}\right) t^{N-m-r-1} \\
& \left.+\sum_{r=0}^{n-k-1}\left(\begin{array}{c}
n-k-1 \\
r
\end{array}\right)(-1)^{r} t^{N-m-r-1} \int_{t_{0}}^{t} s^{m+r} R_{N}(s) d s\right] \\
& \left.-a_{0}^{(k)} t^{N}-a_{1}^{(k)} t^{N-1}-\cdots-a_{N-1}^{(k)} t\right\} \\
& =b_{N} /(m+1-N)(m+2-N) \cdots(m+n-k-N)=a_{N}^{(k)} .
\end{aligned}
$$

The next to the last equality is a result of the facts that $m>N-1$ and

$$
\lim _{t \rightarrow \infty} t^{N-1-m-r} \int_{t}^{t} s^{r+m} R_{N}(s) d s=\lim _{t \rightarrow \infty} \frac{t^{N} R_{N}(t)}{m+r+1-N}=0 .
$$

Thus, the induction is completed and the limit in (6) is equal to $a_{N}^{(k)}$ as given by (11) evaluated at $j=N$. Therefore, we have exhibited the asymptotic expansion of the solution $u(t)$ of (1). This completes the proof of the theorem.

We observe that as a consequence of the expression for the $a_{j}^{(\boldsymbol{k})}$ that $a_{j}^{(k)}=0$ if and only if $b_{j}=0$.

\section{Bibliography}

1. A. Erdelyi, Asymptotic expansions, Dover, New York, 1956.

2. J. K. Hale and N. Onuchic, On the asymptotic behavior of solutions of a class of differential equations, Contributions to Differential Equations 2 (1963), 61-75. 
3. T. G. Hallam, Asymptotic behavior of the solutions of an nth order nonhomogeneous ordinary differential equation, Trans. Amer. Math. Soc. 122 (1966), 177-194.

4. - Asymptotic behavior of the solutions of a nonhomogeneous singular equation, J. Differential Equations 3 (1967), 135-152.

5. E. Hille, Nonoscillation theorems, Trans. Amer. Math. Soc. 64 (1948), 234-252.

6. R. A. Moore and Z. Nehari, Nonoscillation theorems for a class of nonlinear differential equations, Trans. Amer. Math. Soc. 93 (1959), 30-52.

7. P. Waltman, On the asymptotic behavior of a nonlinear equation, Proc. Amer. Math. Soc. 15 (1964), 918-923.

8. - On the asymptotic behavior of solution of an nth order equation, Monatsch. Math. 69 (1965), 427-430.

Florida State University 